\title{
地铁司机室噪声与钢轨波磨关系的试验与仿真研究*
}

\author{
郭建强 ${ }^{1}$ 朱雷威 ${ }^{1}$ 刘晓龙 ${ }^{2}$ 韩 健 ${ }^{2}$ 肖新标 $^{2}$ \\ (1. 中车青岛四方机车车辆股份有限公司 青岛 266000; \\ 2. 西南交通大学牵引动力国家重点实验室 成都 610031)
}

\begin{abstract}
摘要: 地铁司机室的声学环境对于司机的身心健康以及行车安全至关重要。司机室处于在转向架上方, 其噪声主要来源于轮 轨噪声, 而钢轨波磨是地铁中常见的问题, 严重的波磨会显著增大轮轨噪声, 从而影响司机室的声学环境。众所周知波磨与 车内噪声存在正相关的定性关系, 但两者之间的定量关系尚不明确。因此深入地研究钢轨波磨与司机室车内噪声之间的关系, 对司机室车内噪声控制至关重要。本文采取了现场试验与仿真计算相结合的研究方法, 分析了有无波磨对司机室车内噪声的 影响, 以及司机室车内噪声与钢轨波磨之间的定量关系。
\end{abstract}

关键词: 地铁司机室; 车内噪声; 轮轨噪声; 钢轨波磨

中图分类号: U231; TB53

\section{Experimental and Simulation Study on the Relationship Between Interior Noise of Metro Cab and Rail Corrugation}

\author{
GUO Jianqiang ${ }^{1}$ ZHU Leiwei ${ }^{1} \quad$ LIU Xiaolong $^{2} \quad$ HAN Jian $^{2} \quad$ XIAO Xinbiao $^{2}$ \\ (1. CRRC Qingdao Sifang Co. Ltd, Qingdao 266000; \\ 2. State Key Laboratory of Traction Power, Southwest Jiaotong University, Chengdu 610031)
}

\begin{abstract}
The acoustic environment of the metro cab is very important for driver's health and even traffic safety. The main source of the interior noise of the $\mathrm{cab}$ is the wheel/rail noise, since the location of the cab is just above the bogie. Furthermore, the serious rail corrugation is very common in the metro system, which will enhance the wheel/rail noise and have a great effect on interior noise of the cab. As we all know, there is a positive correlation between the interior noise and the rail corrugation, but the quantitative relation is unclear yet. So, it is of great significance for the interior noise control to have an insight in the relationship between interior noise and rail corrugation. A combination method of field test and simulation is adopted in this study to analyze the effect on interior noise caused by rail corrugation and the quantitative relation between them.
\end{abstract}

Key words: metro cab; interior noise; wheel/rail noise; rail corrugation

\section{0 前言}

地铁以其安全准时、载客量大以及节能减排等 优点在世界各大中城市得以迅速发展, 然而地铁振 动噪声问题却严重困扰着乘客以及相关从业人员。 我国地铁运行速度一般在 $60 \sim 120 \mathrm{~km} / \mathrm{h}$, 轮轨噪声 [1-3] 是最为主要的声源, 而轮轨噪声主要是由于车轮 和钢轨表面的粗粘度引起轮轨系统剧烈振动, 从而 辐射噪声。由于地铁运行密度大, 线路条件(小半径 曲线、轨道类型及扣件类型等)复杂, 使得轮轨相互

\footnotetext{
* 国家重点研发计划(2017YFB1201103/2016YFE0205200)和国家自然科 学基金(U1434201/51475390)资助项目。20181002 收到初稿, 20190615 收到修改稿
}

作用加剧, 钢轨波磨现象普遍。GRASSIE 等 ${ }^{[4-6]}$ 研 究了钢轨波磨的特征、产生机理、测试方法以及一 些处理措施; JIN 等 ${ }^{[7-9]}$ 通过建立数值仿真模型, 研 究了曲线钢轨擦伤、离散支撑以及轨道不平顺对钢 轨波磨产生及发展的影响。温泽峰、李霞等 ${ }^{[10-12]}$ 在此基础上进一步建立了较为完整的钢轨波磨理 论计算模型, 揭示了钢轨波磨形成机理, 并提出相 关缓解措施。上述学者深入全面的研究了钢轨波磨 产生机理及影响因素, 为进一步研究钢轨波磨对列 车振动噪声的影响提供了基础。刘维宁等 ${ }^{[13]}$ 通过对 北京某地铁异常波磨的调查, 发现钢轨波磨严重区 段, 车内噪声也异常显著。钢轨打磨是控制钢轨波 磨水平的有效措施, ZHAO 等 ${ }^{[14]}$ 研究表明, 对钢轨 进行打磨可以使车内噪声降低 $3 \sim 4 \mathrm{dBA} ; \mathrm{HAN}^{[15]}$ 
等研究了地铁车内噪声与钢轨波磨之间的关系, 并 在此基础上提出了基于车内噪声的钢轨波磨控制 方法。

大多数研究人员都把研究地铁客室车内噪声作 为研究重点, 而忽视了噪声对司机的影响, 相比于 客室而言, 司机室空间狭小, 并且处于转向架正上 方, 声学环境更为恶劣, 驾驶员长期暴露于该环境 中, 容易产生疲劳和烦恼情绪, 严重威胁行车安全。 本文根据某地铁司机室现场测试数据, 分析了司机 室车内噪声与钢轨波磨之间的关系, 并基于有限 元 边界元方法, 初步研究了司机室车内噪声与钢 轨波磨的定量关系。

\section{1 钢轨波磨对司机室车内噪声影响的 试验}

本文为了调查分析地铁司机室车内噪声与钢轨 波磨之间的关系, 参考标准 ISO $3381^{[16]}$ 的相关规 定，在列车司机室座椅旁与司机人耳平齐的位置布 置了声学测点, 模拟测试司机坐姿耳部位置处的声 压水平，如图 1 所示。同时，在转向架区域关键部 件处布置了加速度传感器和传声器、车体表面布置 了表面声传声器, 用以监测各关键部件的振动、轮 轨噪声以及车体表面噪声, 分析列车司机室车内噪 声与车外振动噪声的关系。针波磨较为严重的区间 进行了钢轨粗粘度测试，并测试了相同区间打磨后 的钢轨粗粘度。如图 2a 所示。试验前一周对列车进 行了车轮锁修, 保证了车轮表面粗糙度较平顺, 消 除过大车轮粗粘度对司机室车内噪声的影响, 如图 $2 b$ 所示。

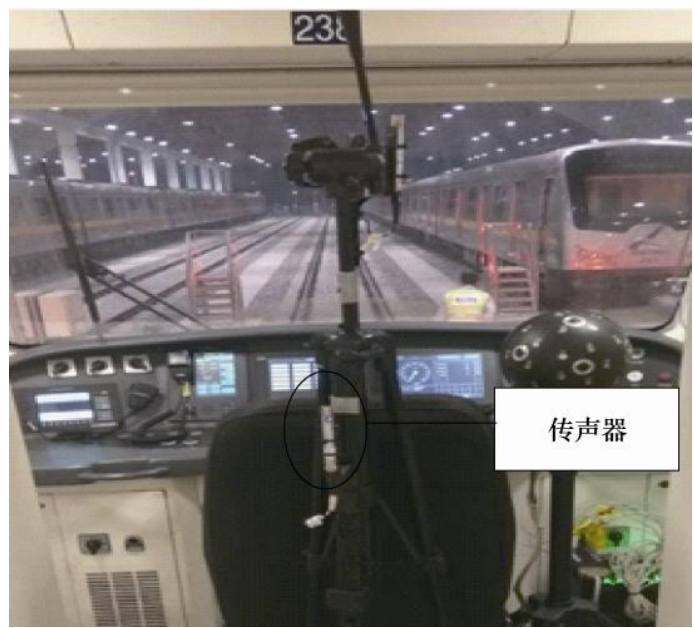

图 1 司机室车内噪声测点

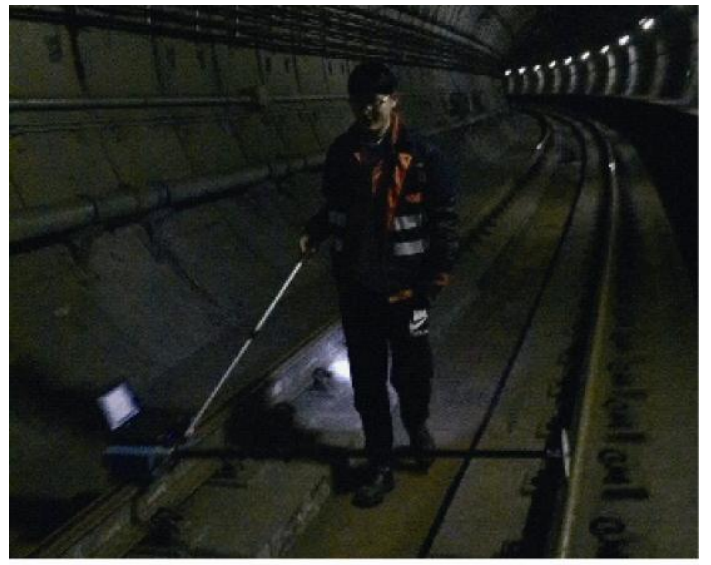

(a) 钢轨波磨测试

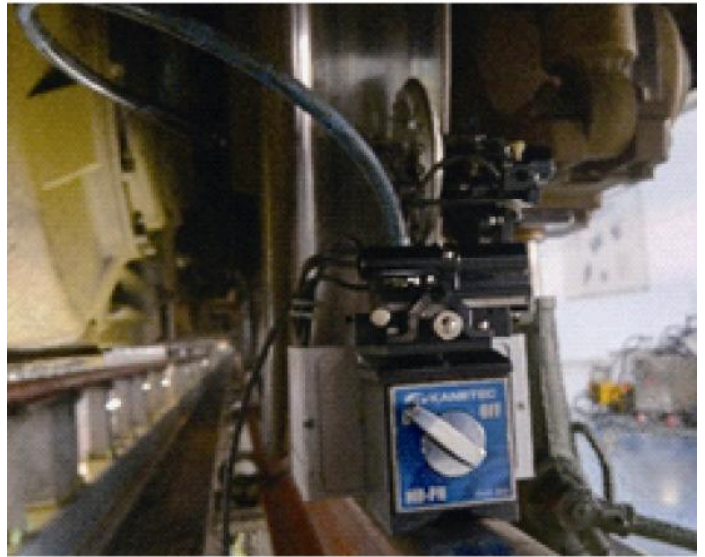

(b) 车轮多边形测试

图 2 轮轨表面粗糙度测试

图 3 给出了列车在钢轨打磨前后以 $55 \mathrm{~km} / \mathrm{h} \pm 5 \%$ 的速度运行于该线路, 司机室车内噪声的总体水平。 由图可知, 列车运行于打磨前各区间时, 司机室车内 噪声普遍高于列车运行于打磨后的相应区间, 平均高 出约 $3.6 \mathrm{~dB}(\mathrm{~A})$, 最大差值约为 $6.7 \mathrm{~dB}(\mathrm{~A})$ 。而两次测 试的车辆相同, 且试验前刚进行了车轮锁修, 车轮表 面状态良好，如图 4 所示，由此可以判断造成两次测 试司机室车内噪声差异的主要原因是钢轨波磨。

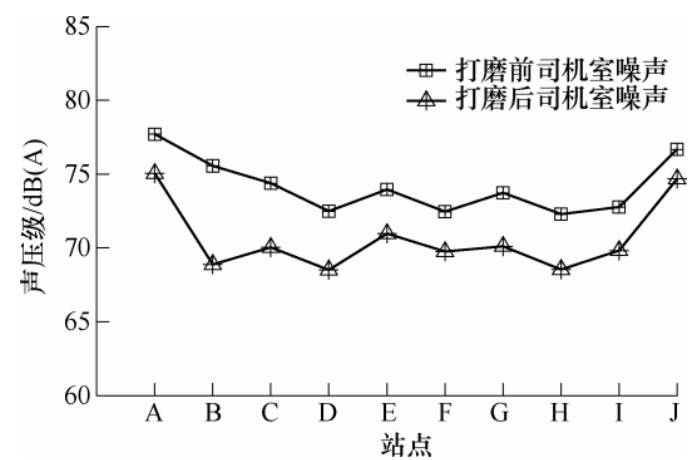

图 3 司机室车内噪声整体概况

选取区间 B 段的数据进行分析。图 5 给出了列 车分别运行于区间 B 段打磨前、后两次测试时列车 的运行速度, 以及车内噪声随里程的变化。 


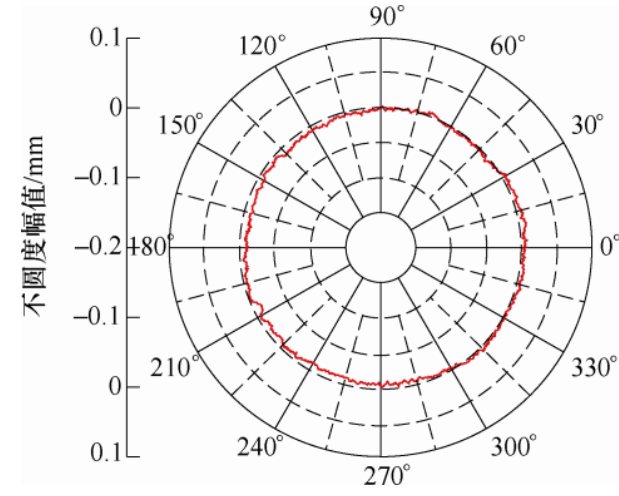

图 4 车轮不圆度测试结果

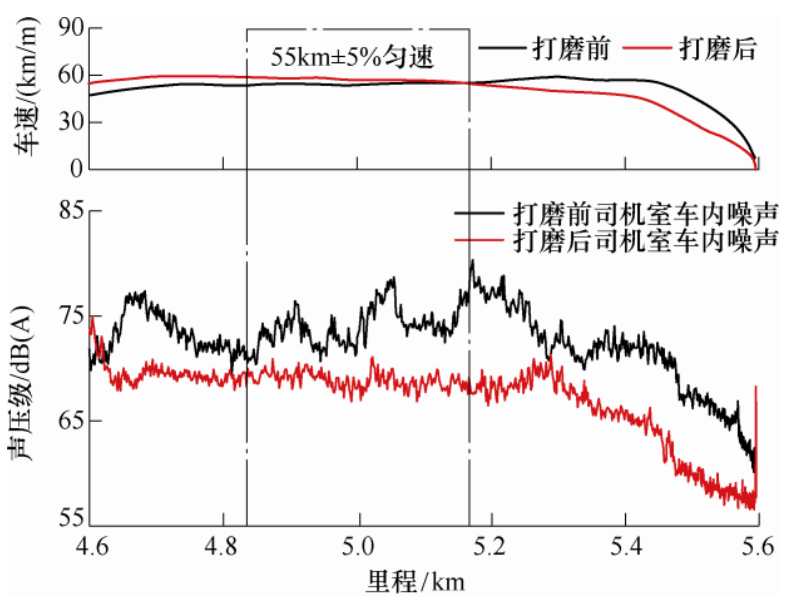

图 5 区间 B 段列车速度及司机室车内噪声

由图 5 可知, 司机室车内噪声与列车运行速度 之间总体存在正相关关系, 当以匀速运行时, 打磨 前后司机室车内噪声差异显著, 最大差异可达到近 $10 \mathrm{~dB}(\mathrm{~A})$ 。截取图 5 中蓝色虚线框内的匀速段数据 进行频谱分析, 如图 6 所示, 给出了该匀速区段司 机室车内噪声三分之一倍频程谱图, 由图 6 可知, 司机室车内噪声最显著的频段分布在 $315 \sim 1000$ $\mathrm{Hz}$ 倍频程带, 而打磨前后车内噪声的显著差异 (315 630 Hz)也在该频段中。研究表明, 500 2 000 $\mathrm{Hz}$ 频段内的铁路噪声主要来源于轮轨噪声的贡献 ${ }^{[15]}$, 因此, 图 7 进一步给出了该区段司机室车内噪声与 轮轨噪声之间关系的频谱图。

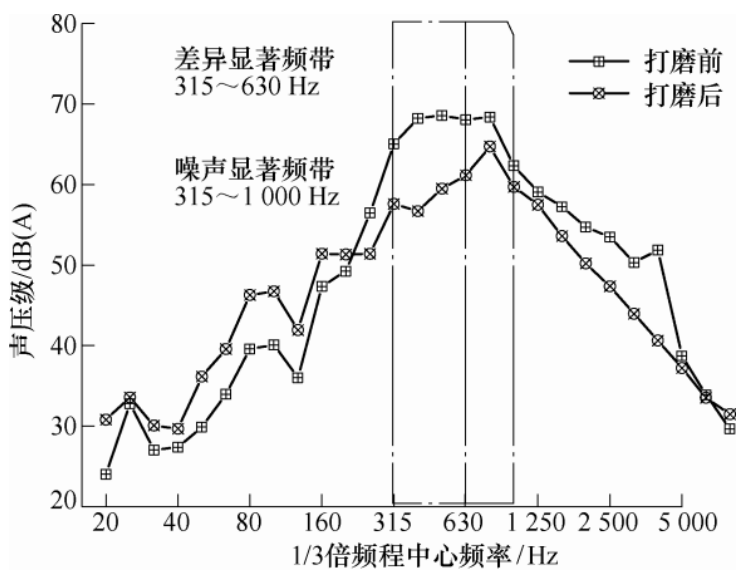

图 6 区间 B 段司机室车内噪声 $1 / 3$ 倍频程谱图
由图 7 可知, 司机室车内噪声显著的频率分别 为 $441 \mathrm{~Hz} 、 359 \mathrm{~Hz}$ 以及 $295 \mathrm{~Hz}$ ，而这三个频率下的 轮轨噪声和车外噪声也最为显著, 即车内噪声显著 频率与轮轨噪声显著频率一一对应; 由此可知, 轮 轨噪声是影响司机室车内噪声的主要原因, 且可以 通过空气路径传入车内。

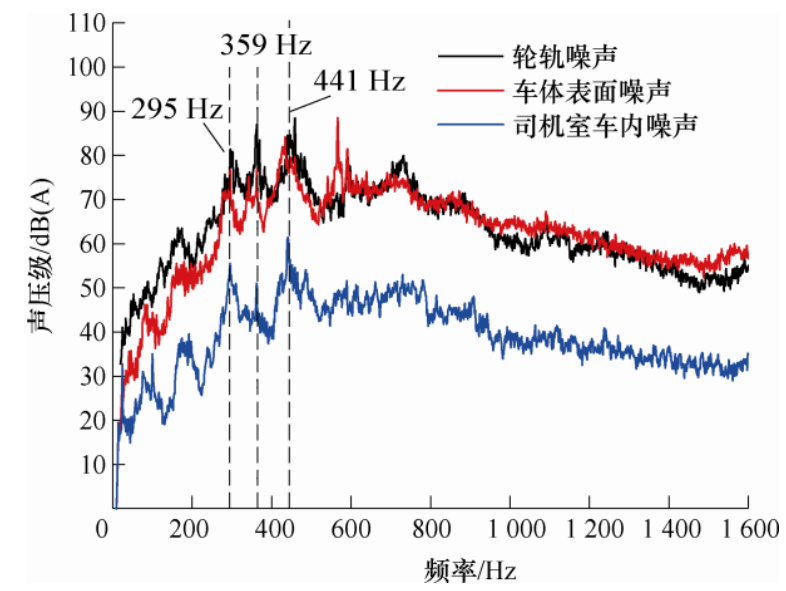

图 7 司机室车内噪声与轮轨噪声频谱图

图 8 又给出了列车运行于该段打磨前后轴箱的 振动加速度频谱图以及车内噪声频谱图, 由图 8 可 知, 轴箱振动加速度幅值显著的频率处, 司机室车 内噪声也最为显著, 由此可见, 轮轨振动也可以通 过结构传递路径传入车内, 进而对车内噪声产生影 响。综上所述, 轮轨系统的相互作用形成的高频振 动声辐射是影响司机室车内噪声的原因。

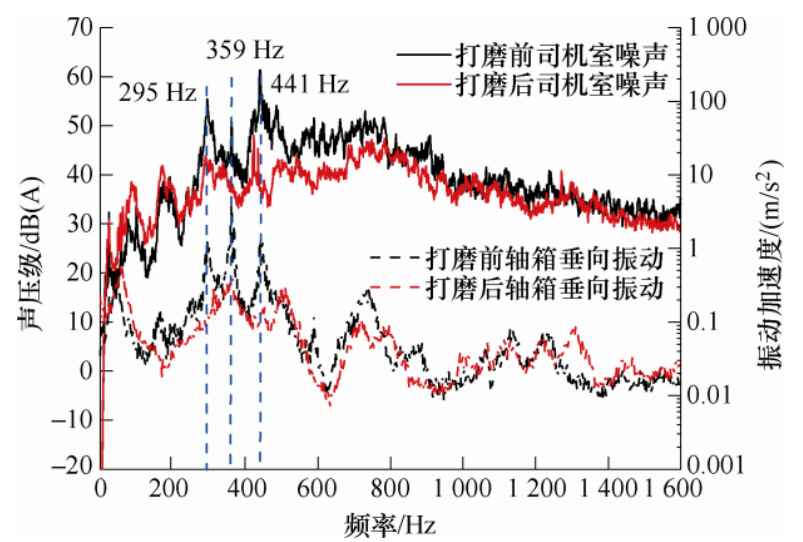

图 8 司机室车内噪声与轴箱振动加速度频谱图

图 9 给出了该区段打磨前后钢轨波磨的测试结 果。由图 9 可知, 该段钢轨打磨前存在特征波长为 30 50 mm 钢轨波磨, 而打磨后该波长范围内的波 磨被明显消除。根据列车运行速度、波磨波长以及 激励频率之间的关系(式(1))可知, 车辆轴箱振动在 $295 \mathrm{~Hz}$ 的显著频率对应波磨波长约为 $50 \mathrm{~mm}$; 359 $\mathrm{Hz}$ 的显著频率对应波磨波长约为 $40 \mathrm{~mm} ; 441 \mathrm{~Hz}$ 的显著频率对应波磨波长约为 $36 \mathrm{~mm}$; 这是导致 $500 \sim 630 \mathrm{~Hz}$ 频率范围内打磨前和打磨后差异的主 
要原因。

$$
f=\frac{v / 3.6}{\lambda / 1000}=277.78 \frac{v}{\lambda}
$$

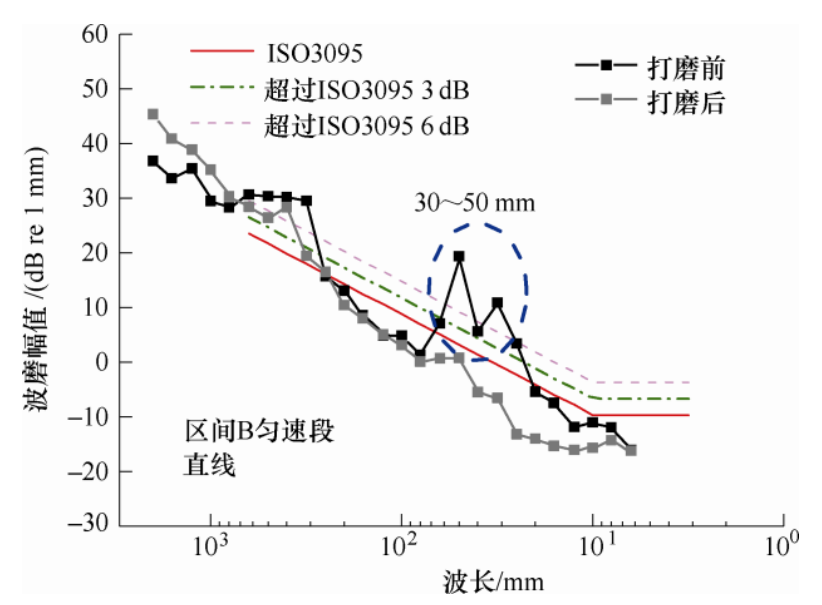

图 9 区间 B 匀速段钢轨波磨

综上所述, 波长为 $30 \sim 50 \mathrm{~mm}$ 的钢轨波磨是引 起司机室车内噪声在 $295 \mathrm{~Hz} 、 359 \mathrm{~Hz}$ 以及 $441 \mathrm{~Hz}$ 频率处噪声异常显著的根本原因。为了进一步研究 钢轨波磨与司机室车内噪声的量化关系, 基于验证 后的仿真模型对司机室车内噪声与钢轨波磨的量化 关系展开分析。

\section{2 钢轨波磨与司机室车内噪声量化关 系的仿真}

为研究钢轨波磨与司机室车内噪声的量化关 系, 本文建立了三维轮轨有限元模型, 如图 10 所示。 轮轨有限元模型中, 车轮采用普通直辐板车轮, 直 径 $840 \mathrm{~mm}$, 由 solid45 实体单元进行离散; 轨道部 分, 采用 UIC60 标准钢轨, 轨枕和道床采用实体单 元进行离散, 扣件系统采用线性的弹簧阻尼单元进 行模拟。垂向刚度为 $80 \times 10^{6} \mathrm{~N} / \mathrm{m}$, 横向刚度为 $50 \times$ $10^{6} \mathrm{~N} / \mathrm{m}$, 纵向刚度为 $40 \times 10^{6} \mathrm{~N} / \mathrm{m}$, 材料参数如表 1 所示。
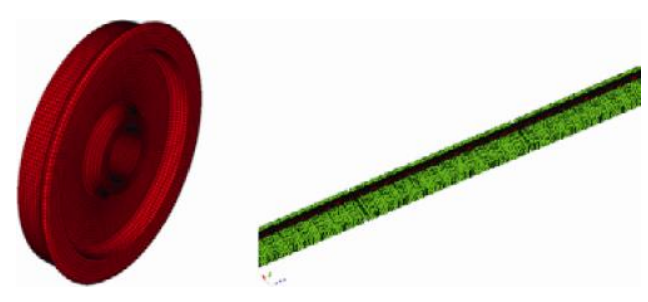

图 10 三维轮轨有限元模型

首先在单位力激励下分别计算出车轮导纳 $\alpha_{W}$ 、 钢轨导纳 $\alpha_{R}$ 与接触导纳 $\alpha_{C}$, 并根据实测轮轨粗粘 度, 考虑接触滤波计算出轮轨联合粗䊁度 $r$, 由式(2)
表 1 模型参数

\begin{tabular}{cccc}
\hline 部件 & 弹性模量/GPa & 泊松比 & 质量 \\
\hline 车轮 & 210 & 0.3 & $7850 \mathrm{~kg} / \mathrm{m}^{3}$ \\
钢轨 & 210 & 0.3 & $60 \mathrm{~kg} / \mathrm{m}$ \\
轨枕 & 35 & 0.2 & $90 \mathrm{~kg}$ \\
轨道板 & 36 & 0.2 & $2500 \mathrm{~kg} / \mathrm{m}^{3}$ \\
\hline
\end{tabular}

求出轮轨相互作用力, 将该轮轨力作为激励输入, 求得轮轨系统振动响应, 再由三维声学积分方程 ${ }^{[15,17]}$ (式(3)) 计算出轮轨噪声, 最后由试验数据建立的轮 轨噪声到司机室内噪声的传递关系, 进一步求出司 机室车内噪声。该传递关系的建立, 需满足两方面 的前提: 轮轨噪声为主要声源; 列车运行环境为混 响场。本次试验中上述条件均满足。

$$
\begin{gathered}
P=r /\left(\alpha_{R}+\alpha_{W}+\alpha_{C}\right) \\
\bar{p}(x, y, z)=-\int_{S}\left(\bar{p}\left(x^{\prime}, y^{\prime}, z^{\prime}\right) \frac{\partial G(R)}{\partial n}+\right. \\
\left.i \rho \omega G(R) v_{n}\left(x^{\prime}, y^{\prime}, z^{\prime}\right)\right) \mathrm{d} S
\end{gathered}
$$

式中, $\bar{p}(x, y, z$ 表示空间某一点的声压幅值, $v_{n}\left(x^{\prime}, y^{\prime}, \mathrm{z}^{\prime}\right)$ 为空气质点沿法向的振动速度, $G(R)$ 为 格林函数。运用三维边界元方法 ${ }^{[17]}$ 求解方程(3)。

图 11 给出了该轮轨噪声预测模型的仿真结果 与现场实测结果的对比图。由图可知, 由仿真计算 得到的轮轨噪声, 与现场试验测得的轮轨噪声在整 个频段内变化规律基本一致, 并且, 在轮轨噪声最 为显著的 $200 \sim 800 \mathrm{~Hz}$ 倍频程带内, 仿真结果与实 测结果吻合得较好。因此, 认为本文所建立的有限 元 边界元模型能够有效地对地铁轮轨噪声进行 预测。

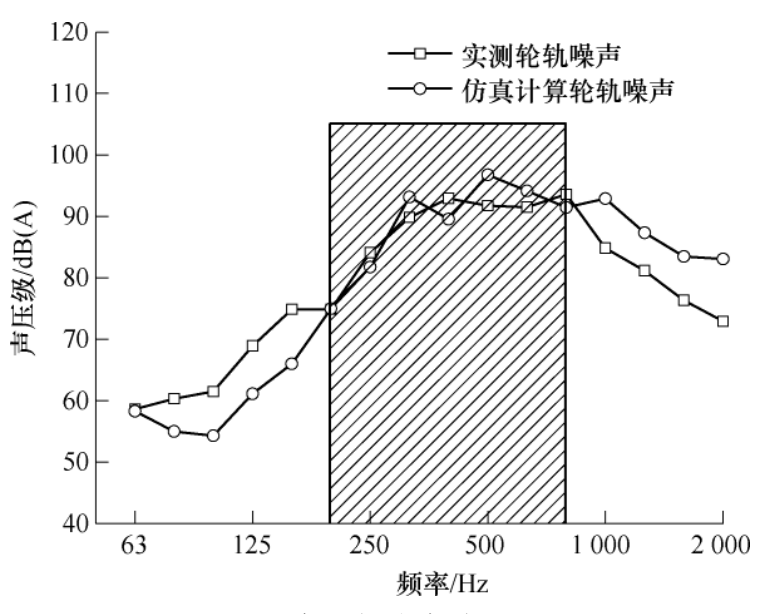

图 11 实测与仿真结果对比

大量研究表明 ${ }^{[10-11]}$, 地铁等城市轨道交通最为 常见的钢轨波磨波长为 $30 \sim 200 \mathrm{~mm}$ 。因此, 为了较 深入全面地研究钢轨波磨与车内噪声之间的量化关 系, 本文将 $30 \sim 200 \mathrm{~mm}$ 分为 $30 \sim 50 \mathrm{~mm} 、 63 \sim 100$ $\mathrm{mm}$ 以及 $125 \sim 200 \mathrm{~mm}$ 三个波长区段, 分别调查各 
个波段波磨幅值在 ISO3095 标准基础上增加若干分 贝, 对车内噪声的影响。

图 12 给出了波长为 $30 \sim 50 \mathrm{~mm}$ 的钢轨波磨幅 值, 分别增加 $2 \mathrm{~dB} 、 3 \mathrm{~d} 、 4 \mathrm{~dB}$ 和 $5 \mathrm{~dB}$ 时, 仿真计 算司机室车内噪声三分之一倍频程与噪声总值, 由 图 12 可知, 增加了特征波长钢轨波磨的粗粘度幅 值, 司机室内特征频率噪声幅值也相应提高, 在 ISO3095 标准钢轨粗粘度谱作用下, 司机室车内噪 声总值为 $78.3 \mathrm{~dB}(\mathrm{~A})$; 当分别增加特征波长粗粘度 幅值 $2 \mathrm{~dB} 、 3 \mathrm{~dB}$ 和 $4 \mathrm{~dB}$ 时, 司机室车内噪声总值 分别提高了 $0.7 \mathrm{~dB}(\mathrm{~A}) 、 1.2 \mathrm{~dB}(\mathrm{~A})$ 和 $1.6 \mathrm{~dB}(\mathrm{~A})$, 当 粗䊁度幅值增加 $5 \mathrm{~dB}$ 时, 车内噪声达到 $80.5 \mathrm{~dB}(\mathrm{~A})$, 提高了 $2.3 \mathrm{~dB}(\mathrm{~A})$, 此时车内噪声已超过 GB14892 ${ }^{[19]}$ 中规定的地铁司机室车内噪声限值。由此可确定针 对该条线路地铁和车辆的基于司机室噪声控制的钢 轨 30 50 mm 波磨的控制限值, 如图 13 所示。

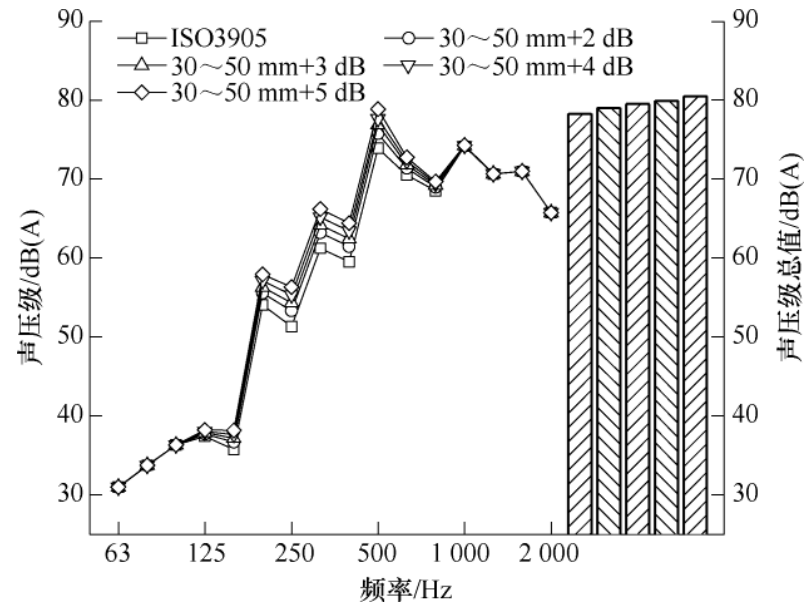

图 12 司机室车内噪声频谱及总值

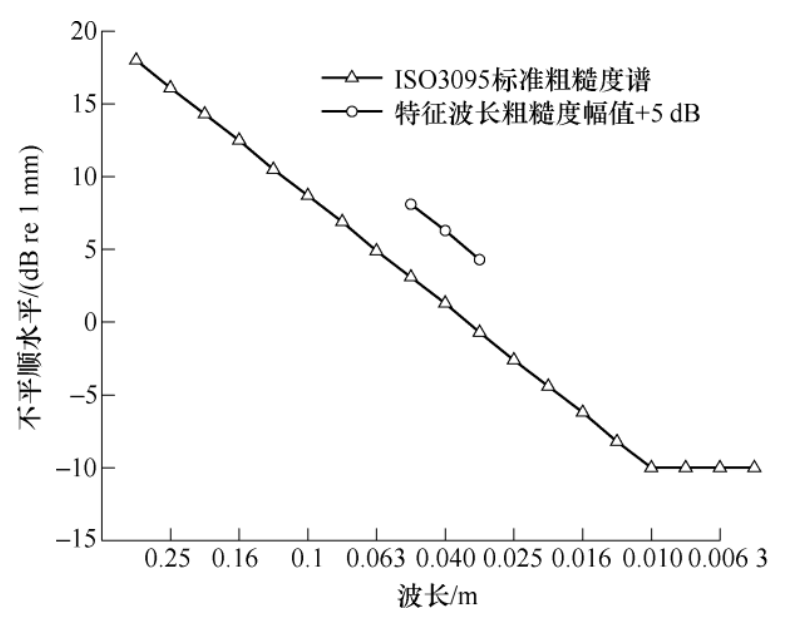

图 $13 \quad 30 \sim 50 \mathrm{~mm}$ 钢轨波磨限值

图 14 给出了波长为 $63 \sim 100 \mathrm{~mm}$ 的钢轨波磨幅 值, 分别增加 $5 \mathrm{~dB} 、 10 \mathrm{~dB} 、 15 \mathrm{~dB}$ 和 $20 \mathrm{~dB}$ 时, 仿 真计算司机室车内噪声三分之一倍频程与噪声总 值, 由图 14 可知, 车内噪声随钢轨波磨幅值变化规
律与上述分析相似。当波长为 $63 \sim 100 \mathrm{~mm}$ 的钢轨 波磨幅值增加时, 响应的噪声频段幅值显著增加, 但是由于该频段不是车内噪声最为显著的频段, 因 此, 车内噪声总值对该频段噪声变化的敏感度较低。 所以波长为 $63 \sim 100 \mathrm{~mm}$ 的钢轨波磨幅值增加约 20 $\mathrm{dB}$ 时, 车内噪声总值增加约 $2.0 \mathrm{~dB}(\mathrm{~A})$, 此时车内 噪声超过限值。由此可确定针对该条线路地铁和车 辆的基于司机室噪声控制的钢轨 $63 \sim 100 \mathrm{~mm}$ 波磨 的控制限值, 如图 15 所示。

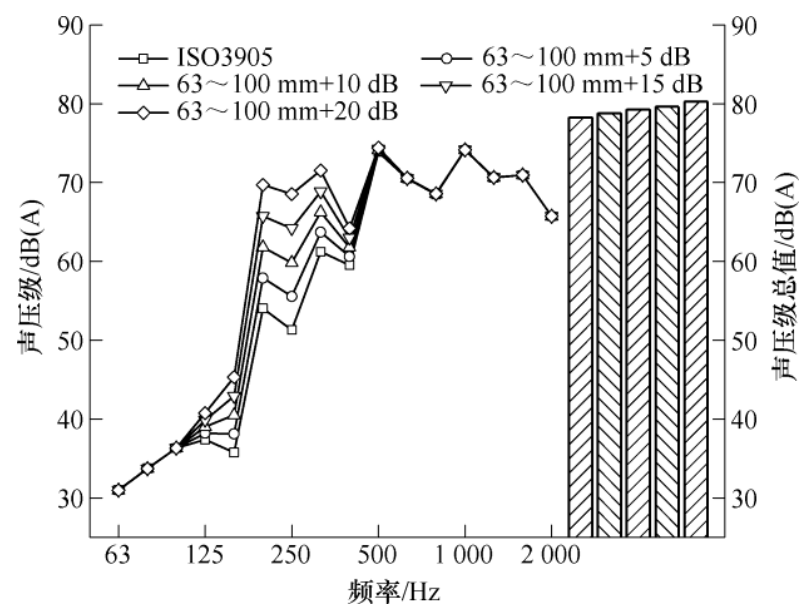

图 14 司机室车内噪声频谱及总值

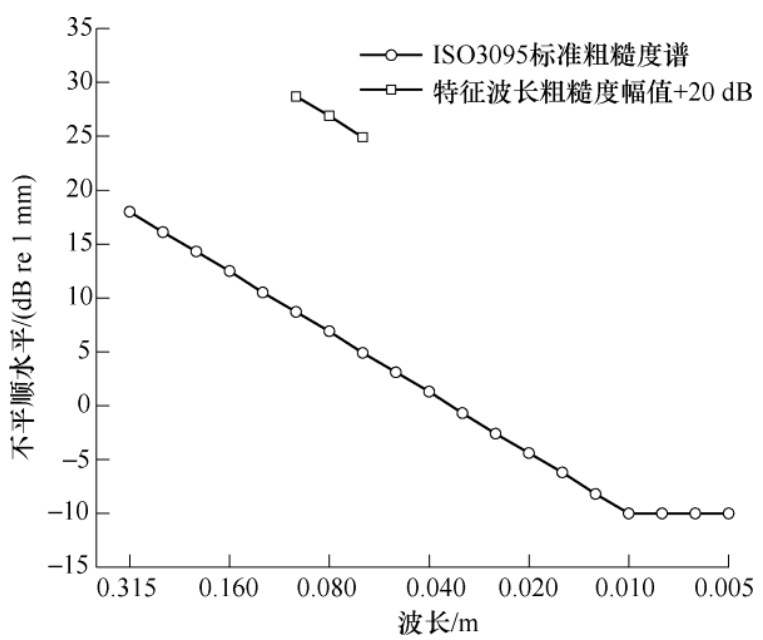

图 $15 \quad 63 \sim 100 \mathrm{~mm}$ 钢轨波磨限值

图 16 给出了波长为 $125 \sim 200 \mathrm{~mm}$ 的钢轨波磨 幅值, 分别增加 $10 \mathrm{~dB} 、 20 \mathrm{~dB}$ 和 $35 \mathrm{~dB}$ 时, 仿真计 算司机室车内噪声三分之一倍频程与噪声总值。波 长为 $125 \sim 200 \mathrm{~mm}$ 的钢轨波磨对应于车内噪声 $80 \sim 200 \mathrm{~Hz}$ 倍频程带, 该频段内噪声幅值随波磨幅 值增加而增加, 由于低频部分车内噪声对噪声总值 贡献非常小，即使增加该对应波长波磨幅值 $30 \mathrm{~dB}$, 车内噪声仅提高了 $0.2 \mathrm{~dB}(\mathrm{~A})$ 。因此, 由于该波长段 钢轨波磨对车内噪声贡献非常小, 因此不再进一步 调查该波长段波磨幅值对车内噪声的影响。 


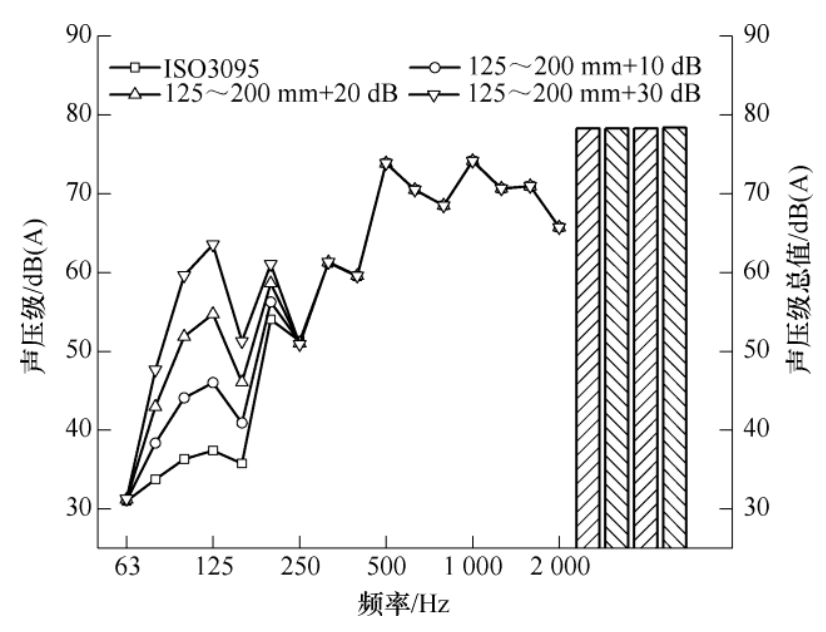

图 16 司机室车内噪声频谱及总值

\section{3 结论}

本文采取了现场试验与仿真计算相结合的研究 方法, 分析了有无波磨对司机室车内噪声的影响, 以及司机室车内噪声与钢轨波磨之间的定量关系。 主要研究结论如下。

(1) 钢轨打磨前, 司机室车内噪声显著的频率 $295 \mathrm{~Hz} 、 359 \mathrm{~Hz}$ 以及 $441 \mathrm{~Hz}$, 与轴箱振动显著的频 率相对应, 该线路主要存在特征波长为 $30 \sim 50 \mathrm{~mm}$ 的钢轨波磨, 在试验运行速度下, 钢轨波磨的激振 频率与列车振动噪声显著频率相吻合, 钢轨波磨是 引起轴箱振动、司机室车内噪声显著的主要原因。 钢轨打磨后, 波长为 $30 \sim 50 \mathrm{~mm}$ 的钢轨波磨被明显 消除, 打磨后的司机室车内噪声比打磨前降低 3.6 $\mathrm{dB}(\mathrm{A})$ 。

(2) 仿真结果表明, 随着钢轨粗糙度幅值的增 加, 司机室车内噪声也随之增加, 波长为 $30 \sim 50 \mathrm{~mm}$ 的钢轨波磨幅值增加 $5 \mathrm{~dB}$ 时, 司机室车内噪声增加 $2.4 \mathrm{~dB}(\mathrm{~A})$, 此时司机室车内噪声达到 $80.5 \mathrm{~dB}(\mathrm{~A})$, 已超过了司机室车内噪声限值; 波长为 $63 \sim 100 \mathrm{~mm}$ 的钢轨波磨幅值增加 $20 \mathrm{~dB}$ 时, 司机室车内噪声增 加 $2.0 \mathrm{~dB}(\mathrm{~A})$, 此时司机室车内噪声达到 $80.1 \mathrm{~dB}(\mathrm{~A})$, 已超过了司机室车内噪声限值。由此可确定针对 该条线路地铁和车辆的基于司机室噪声控制的钢 轨波长为 $30 \sim 50 \mathrm{~mm}$ 和 $63 \sim 100$ 波磨的控制限值; 波长为 $125 \sim 200 \mathrm{~mm}$ 的钢轨波磨对车内噪声影响非 常小。

\section{参 考 文 献}

[1] THOMPSON D J. Railway noise and vibration : Mechanisms, modelling and means of control [M]. Amsterdam: Elsevier, 2009.

[2] 韩健, 肖新标, 金学松, 等. 城市轨道交通车轮振动声
辐射特性[J]. 机械工程学报，2012，48(10)：116-121.

HAN Jian, XIAO Xinbiao, JIN Xuesong, et al. Sound radiation characteristics of wheel used in urban rail traffic[J]. Journal of Mechanical Engineering, 2012, 48(10): 116-121.

[3] HAN Jian, WANG Ruiqian, WANG Di, et al. Effect of wheel load on wheel vibration and sound radiation study on the performance of dynamic vibration absorbers of train wheels $[\mathrm{J}]$. Chinese Journal of Mechanical Engineering, 2015, 28(1): 46-54.

[4] GRASSIE S L, KALOUSEK J. Rail corrugation : Characteristics, causes and treatments[J]. Proceedings of the Institution of Mechanical Engineers, Part F: Journal of Rail and Rapid Transit, 1993，207(1): 57-68.

[5] GRASSIE S L. Short wavelength rail corrugation: Field trials and measuring technology[J]. Wear, 1996, 191(1-2): 149-160.

[6] GRASSIE S L. Rail corrugation: Characteristics, causes, and treatments $[\mathrm{J}]$. Proceedings of the Institution of Mechanical Engineers, Part F: Journal of Rail and Rapid Transit, 2009, 223(6): 581-596.

[7] JIN Xuesong, WEN Zefeng, WANG Kaiyun, et al. Effect of a scratch on curved rail on initiation and evolution of rail corrugation[J]. Tribology International, 2004, 37: 385-94.

[8] JIN Xuesong, WANG Kaiyun, WEN Zefeng, et al. Effect of discrete supports of rail on initiation and evolution of rail corrugation $[\mathrm{J}]$. Chinese Journal of Mechanical Engineering, 2005, 18: 37-41.

[9] JIN Xuesong, WEN Zefeng, WANG Kaiyun. Effect of track irregularities on initiation and evolution of rail corrugation. Journal of Sound and Vibration，2005，285: $121-48$.

[10] 温泽峰. 钢轨波浪形磨损研究[D]. 成都: 西南交通大 学, 2006 .

WEN Zefeng. Study on rail corrugation[D]. Chengdu: Southwest Jitong University， 2006.

[11] 李霞. 地铁钢轨波磨形成机理研究[D]. 成都: 西南交 通大学, 2012.

LI Xia. Study on the mechanism of railway corrugation on subway track[D]. Chengdu: Southwest Jitong University, 2012.

[12] LI Xia, LI Wei, WANG Hengyu. Study on the mechanism of rail corrugation of subway track with vibration-absorbing fasteners[C]//Proceedings of the 9 th International Conference on Contact Mechanics and Wear of Rail/Wheel Systems， 2012: 205-215.

[13] 刘维宁, 任静, 刘卫丰, 等. 北京地铁钢轨波磨测试分 析[J]. 都市快轨交通，2011，24(3)：6-9. 
LIU Weining, REN Jing, LIU Weifeng, et al. In-situ tests and analysis on rail corrugation of Beijing Metro[J]. Urban Rapid Rail Transit, 2011，24(3): 6-9.

[14] ZHAO C Y, WANG P, YI Q. Internal noise reduction in railway vehicles by means of rail grinding and rail dampers [J]. Noise Control Engineering Journal, 2017, 65(1): 1-13.

[15] HAN Jian, XIAO Xinbiao, WU Yue, et al. Effect of rail corrugation on metro interior noise and its control [J]. Applied Acoustics, 2018, 130: 63-70.

[16] International Organization for Standardization ISO 3381-2011, Railway applications-Acoustics-Measurement of noise inside railbound vehicles[S]. Switzerland: ISO Technical Committees, 2011.

[17] WU T W. Boundary element acoustics[M]. Southampton: WIT Press, 2005.

[18] 李伟, 温泽峰, 王衡禹, 等. 地铁钢轨波磨演化过程中
的特征分析 [J]. 机械工程学报, 2018, 54(4): 70-78.

LI Wei, WEN Zefeng, WANG Hengyu, et al. Analysis on evolution characteristics of rail corrugation on a metro[J]. Journal of Mechanical Engineering. 2018， 54(4): 70-78.

[19] 中华人民共和国国家标准(GB 14892-2006). 城市轨道 交通列车噪声限值和测量方法 $[S]$. 北京: 中国国家标注 化委员会, 2006 .

National Standard of the People's Republic of China (GB 14892-2006). Noise limit and measurement for train of urban rail transit [S]. Beijing: National Standardization Co mmittee of the People's Republic of China, 2006.

作者简介: 郭建强, 男, 1980 年出生, 博士。主要研究方向为轨道车辆 噪声控制。

E-mail: 15066280095@163.com

肖新标(通信作者), 男, 1978 年出生, 博士, 副研究员, 硕士研究生导 师。主要研究方向为列车振动噪声分析与控制。

E-mail: xiao@home.swjtu.edu.cn 